\title{
Seleção para produção de leite em núcleos MOET das raças zebuínas: um estudo de simulação
}

[Selection to milk production in Zebu MOET nuclei: a simulation study]

\author{
M.G.C.D. Peixoto ${ }^{1}$, C.S. Pereira ${ }^{1}$, C.G. Fonseca ${ }^{2}$, F.E. Madalena ${ }^{1}$ \\ ${ }^{1}$ Departamento de Zootecnia - Escola de Veterinária da UFMG \\ Caixa Postal 567 \\ 30123-970, Belo Horizonte, MG \\ ${ }^{2}$ Departamento de Biologia Geral - ICB - UFMG
}

\begin{abstract}
RESUMO
Um programa estocástico de seleção em núcleo MOET, esquema adulto, com geração discreta e população panmítica finita, foi simulado para uma característica influenciada por grande número de locos, mediante um modelo estocástico. Foram consideradas as herdabilidades de 0,25, 0,35 e 0,45 e a repetibilidade de 0,50 . Os fatores utilizados na definição dos esquemas alternativos de núcleo MOET estudados foram: tamanho da população $(\mathrm{N})$, estratégia de acasalamento $(\mathrm{A})$, número de progênies MOET nascidas $(\mathrm{P})$ e número de reprodutores selecionados por grupo de irmãos completos $(\mathrm{R})$. Os tamanhos de população que constituíram a geração base do núcleo foram: 16 doadoras/ 8 reprodutores; 16 doadoras $/ 4$ reprodutores e 32 doadoras/4 reprodutores. Na estratégia hierárquica $(\mathrm{H})$ de acasalamento, cada doadora foi acasalada com apenas um reprodutor, enquanto na estratégia fatorial de acasalamento, cada doadora foi acasalada com dois (F-I) ou quatro (F-II) reprodutores por geração. Considerou-se o nascimento de quatro ou oito progênies MOET por doadora a cada superovulação. O número de reprodutores selecionados foi limitado a um ou dois de cada grupo de irmãos completos. Para as três estratégias de acasalamento (H, F-I e F-II), foi utilizado o intervalo de gerações (IG) de 3,89 anos e, para a estratégia FII, o IG de 4,15 anos. Todos os fatores utilizados na definição dos esquemas alternativos influenciaram $(\mathrm{P}<0,05)$ os resultados do ganho genético nas três herdabilidades consideradas. $\mathrm{O}$ efeito de algumas interações ( $N \times A, N \times R$ e $P \times A)$ também foi significativo $(P<0,05)$. A interação $A \times R$ influenciou $(P<0,05)$ apenas nas herdabilidades de 0,25 e 0,35 . Para a taxa de endogamia, verificou-se influência $(P<0,05)$ de todos os fatores estudados para as três herdabilidades. $\mathrm{O}$ efeito das interações $\mathrm{N} \times \mathrm{P}, \mathrm{N} \times \mathrm{A}, \mathrm{N} \times \mathrm{R}$ e $\mathrm{P} \times \mathrm{A}$ também foi significativo $(P<0,05)$ sobre a taxa de endogamia. Verificou-se que a eficiência do MOET em produzir estruturas viáveis e prenhezes positivas, bem como o grau de redução no IG, constituem os principais aspectos responsáveis pela taxa de progresso genético nos esquemas de seleção em núcleos MOET.
\end{abstract}

Palavras-chave: núcleo MOET, ganho genético, endogamia, simulação

\begin{abstract}
A stochastic genetic simulation of adult MOET breeding nucleus schemes was carried out. A finite panmitic population with discrete generation and selection for a trait under an infinitesimal model were assumed. The heritabilities of $.25, .35$ and .45 and the repeatability of .50 were considered. Different population sizes $(N)$, mating strategies $(A)$, number of MOET progenies $(P)$ and number of sires selected from full sib family $(R)$ used in the definition of the nucleus scheme were evaluated. Population sizes were of 16 donors/8 sires, 16 donors/4 sires and 32 donors/4 sires. Mating strategies were hierarchical (H) each donor was mated to only one sire, factorial I (F-I) - each donor was mated to two sires, and
\end{abstract}

Recebido para publicação em 10 de fevereiro de 2004

Recebido para publicação, após modificações, em 29 de setembro de 2004

E-mail: mgabycdp@icb.ufmg.br 
factorial II (F-II) - each donor was mated to four sires. The number of MOET progenies from each donor by superovulation was 4 or 8. The number of selected sires from each full sib family was 1 or 2. Generation interval (IG) of 3.89 years was used whatever the mating strategy evaluated and the IG of 4.15 was also used when the F-II mating strategy was evaluated. All factors evaluated affected significantly $(P<0.05)$ the genetic gain for all heritabilities considered. Interactions between $N \times A, N \times R$ and $P \times A$ were also significant $(P<0.05)$. The interaction $A \times R$ had a significant effect $(P<0.05)$ only for heritabilities of .25 and .35 . All factors studied for all heritability values had a significant effect $(P<0.05)$ on the inbreeding rate. The $N \times P, N \times A, N \times R$ and $P \times A$ interactions had also a significant effect $(P<0.05)$ on the inbreeding rate. It was strengthened that the MOET efficiency in producing viable embryos and positive pregnancies, besides the degree of reduction in $I G$, are responsible for the genetic progress rate in the MOET selection nucleus schemes.

Keywords: MOET nucleus, genetic gain, inbreeding, simulation

\section{INTRODUÇÃO}

Muitos esforços têm sido concentrados na busca de alternativas que possibilitem maximizar a taxa de resposta à seleção no melhoramento genético do rebanho leiteiro nacional adaptado às condições tropicais. Dentre elas, encontram-se os núcleos de melhoramento genético, que consistem em rebanhos estabelecidos a partir de animais geneticamente superiores de uma determinada raça, nos quais os esforços de teste $\mathrm{e}$ a seleção de animais para uma ou mais características de interesse estariam concentrados. Os núcleos de melhoramento, segundo Smith (1960), têm apresentado boas perspectivas para a obtenção de animais melhoradores.

De acordo com Hinks (1978), os núcleos de melhoramento surgiram da necessidade de procedimentos alternativos que suportassem as mudanças nas condições de produção e mercado, promovessem auto-suficiência nos países com pouca infra-estrutura para executar programas de melhoramentos eficazes e extensos e assegurassem controle mais direto dos fatores determinantes da mudança genética. Os núcleos vêm sendo utilizados há vários anos para o melhoramento genético de suínos, ovinos e bovinos (Hinks, 1978) e, segundo o autor, seu mérito constitui alternativa de melhoramento genético. Entretanto, foi a partir do desenvolvimento da técnica de ovulação múltipla e da transferência de embriões (MOET) que seu potencial pôde ser definido (Nicholas e Smith, 1983; Seidel Jr., 1984).

Os núcleos MOET de melhoramento consistem em rebanhos que utilizam a técnica de ovulação múltipla seguida de transferência de embriões para constituir, rapidamente, amplas famílias de irmãos completos e meio-irmãos a serem avaliadas quanto ao mérito genético. A seleção nos núcleos, segundo Nicholas e Smith (1983), baseia-se nos índices de família, nos quais estão combinadas as informações dos ancestrais (pais e avós) e dos colaterais (irmãos completos e meioirmãos). Assim, o intervalo de gerações seria reduzido, e a taxa de progresso genético seria aumentada, compensando a menor acurácia da seleção com base no índice de família em relação à seleção com base no teste de progênie, principalmente se as herdabilidades para as características sob seleção forem baixas (Smith, 1988; Falconer, 1989; Ruane, 1991).

Os esquemas de melhoramento em núcleos MOET vêm, desde então, sendo bastante estudados e alguns já foram implantados na Inglaterra, França, Dinamarca (Ruane, 1988) e no Brasil (Penna et al., 1998). Apesar dos poucos resultados práticos, os resultados teóricos têm se mostrado bastante promissores (Ruane e Thompson, 1991; Strandén et al., 1991). O salto genético inicial, devido à seleção de animais para constituírem a população-base, é considerado, no entanto, o principal responsável pela superioridade no mérito genético na população do núcleo (Woolliams e Wilmut, 1989; Ruane e Thompson, 1991).

Alguns autores alertaram para o fato de que a utilização das informações de família para seleção de animais melhoradores, aliada ao pequeno tamanho do núcleo, reduz o tamanho efetivo da população, pois aumenta a probabilidade de seleção de indivíduos de uma mesma família, podendo levar ao acréscimo no 
coeficiente médio de endogamia da população (Woolliams, 1989; Keller et al., 1990; Toro et al., 1991). Segundo Keller et al. (1990), o aumento no coeficiente de endogamia será tanto maior, quanto maiores a herdabilidade da característica e a intensidade de seleção aplicada.

Diversos fatores foram apontados como causas de variação no ganho genético e na taxa de endogamia nesses esquemas e, portanto, vêm sendo estudados para auxiliar na definição de alternativas de seleção em núcleos MOET (Nicholas e Smith, 1983; Woolliams e Wilmut, 1989; Ruane e Thompson, 1991). O presente estudo teve os objetivos de comparar o ganho genético possível e avaliar o efeito de cada fator e suas interações em esquemas alternativos de seleção em núcleo MOET adulto.

\section{MATERIAL E MÉTODOS}

Um programa contínuo de seleção foi simulado para uma característica influenciada por grande número de locos, em núcleo MOET de melhoramento, esquema Adulto (Nicholas e Smith, 1983), com gerações discretas.

No programa de simulação utilizado, a seleção é para uma característica; os valores gênicos são calculados pelo modelo animal; a endogamia é considerada na geração dos dados e nas avaliações genéticas; o número de acasalamentos por fêmea pode ser mudado; o número de rebanhos pode ser especificado; a herdabilidade e a repetibilidade podem ser mudadas; e os rebanhos podem ter diferente número de vacas (Strandén et al., 1991).

Quatro fatores foram utilizados na definição dos esquemas de núcleo a serem estudados: tamanho do núcleo, estratégia de acasalamento, número de progênies MOET nascidas e número de reprodutores selecionados de cada grupo de irmãos completos. $\mathrm{O}$ tamanho do núcleo consistiu em modificações no número de animais e na proporção de doadoras por reprodutor. Foram avaliados esquemas com 16 doadoras e oito reprodutores (duas doadoras por reprodutor), 16 doadoras e quatro reprodutores (quatro doadoras por reprodutor) e 32 doadoras e quatro reprodutores (oito doadoras por reprodutor).
As estratégias de acasalamento abrangeram o modelo hierárquico $(\mathrm{H})$, no qual cada fêmea foi acasalada a apenas um reprodutor, originando grupos de irmãos completos e de meio-irmãos paternos; o modelo fatorial I (F-I), no qual cada fêmea foi acasalada a dois reprodutores, originando grupos de irmãos completos, meioirmãos paternos e meio-irmãos maternos; e o modelo fatorial II (F-II), no qual cada fêmea foi acasalada a quatro reprodutores, originando os grupos de irmãos descritos para F-I. Para o número de progênies MOET nascidas, foram assumidos os valores de quatro ou oito. Para o número de reprodutores selecionados de cada grupo de irmãos completos, foram considerados os valores de um ou dois.

Na Tab. 1 são apresentados os fatores utilizados na definição dos esquemas alternativos de núcleo. Os valores e as estratégias estudados para cada fator foram estabelecidos de acordo com a viabilidade financeira de implantação de uma população em núcleo MOET, de acordo com os resultados que até então vêm sendo obtidos no MOET para as raças zebuínas (Coelho et al., 1988; Coelho e Azevedo, 1991) e de acordo com os métodos de acasalamento e de seleção descritos na literatura (Falconer, 1989). A taxa reprodutiva foi ignorada neste programa e foi assumido o número constante de progênies MOET nascidas por doadora.

As mudanças no tamanho da população, no número de progênies MOET nascidas e no número de touros selecionados por grupo de irmãos completos permitiram avaliar as mudanças na intensidade de seleção e na taxa de endogamia e, conseqüentemente, na taxa de progresso genético em cada sistema de acasalamento.

Os valores dos parâmetros herdabilidade e repetibilidade da produção de leite foram escolhidos com a preocupação de que compreendessem os valores das diversas estimativas obtidas para as raças zebuínas em trabalhos realizados no Brasil. Para a herdabilidade, foram assumidos os valores de $0,25,0,35$ e 0,45 , e, para a repetibilidade, assumiu-se o valor de 0,50 (Madalena, 1988; Lôbo et al., 2000). Para a idade à puberdade, assumiu-se o valor de 15,1 meses (Sacco et al., 1987). 
Tabela 1. Descrição dos esquemas de núcleo em função dos fatores utilizados na sua definição

\begin{tabular}{lccc}
\hline Fator & \multicolumn{2}{c}{ Esquema alternativo de núcleo } \\
\hline Tamanho do núcleo (doadoras/reprodutores): & $16 / 8$ & $16 / 4$ & $32 / 4$ \\
Número de doadoras/reprodutor: & 2 & 4 & 8 \\
Número de progênies MOET/doadora: & 4 ou 8 & 4 ou 8 & 4 ou 8 \\
Número de progênies MOET/doadora/acasalamento: & & & \\
$\quad$ Hierárquico & 4 ou 8 & 4 ou 8 & 4 ou 8 \\
- Fatorial I & 2 ou 4 & 2 ou 4 & 2 ou 4 \\
$\quad$ Fatorial II & 1 ou 2 & 1 ou 2 & 1 ou 2 \\
Número de progênies MOET/reprodutor: & 8 ou 16 & 16 ou 32 & 32 ou 64 \\
Número total de progênies MOET/geração: & 64 ou 128 & 64 ou 128 & 128 ou 256 \\
Proporção de progênies fêmeas selecionadas: & $1 / 2$ ou $1 / 4$ & $1 / 2$ ou $1 / 4$ & $1 / 2$ ou $1 / 4$ \\
Número de progênies machos selecionados/família: & 1 ou 2 & 1 ou 2 & 1 ou 2 \\
\hline
\end{tabular}

O intervalo de gerações (IG) foi obtido como proposto por Strandén et al. (1991), levando em consideração a necessidade de coletas extras para a obtenção do número de embriões requeridos no programa, o que resultou no IG de 3,89 anos. Para os esquemas fatoriais com quatro reprodutores acasalados por doador, foi calculado também um novo intervalo de gerações, 4,15 meses, pressupondo a necessidade de novas coletas para a realização dessa estratégia de acasalamento.

A combinação dos diferentes níveis dos fatores estudados resultou em 36 esquemas alternativos de núcleos MOET. Cada esquema alternativo foi simulado em diferentes níveis de herdabilidade. Foram efetuadas oito gerações discretas de seleção, e o ganho genético anual foi calculado como a média entre a primeira e a oitava gerações de seleção, quando a geração-base foi considerada como a geração "O", e os resultados, fornecidos em unidades de desvio-padrão fenotípico.

Para cada combinação, foram estudadas 10, 20, 50 e 100 replicações para determinar o número de replicações com o qual se obteriam estimativas mais precisas do ganho genético e da taxa de endogamia mediante simulação. Escolheu-se proceder a 50 replicações, uma vez que os resultados obtidos com 100 replicações não diferiram daqueles obtidos com 50 replicações quanto à média e ao desvio-padrão. $\mathrm{O}$ resultado final para cada esquema alternativo foi a média de todas as replicações

Na simulação, gerou-se uma grande população de animais não aparentados, com base nos parâmetros fornecidos, da qual, após seleção por truncamento, obteve-se a geração-base do núcleo. Os animais cujos valores gênicos estavam acima de determinado limite foram aceitos. $\mathrm{O}$ valor limite foi inicialmente estabelecido pelo diferencial de seleção fornecido no input. Uma vez que da população gerada não se obteve o número de animais com a superioridade genética desejada, novos candidatos foram gerados até que o número de animais necessários para se estabelecer o núcleo fosse alcançado. As fêmeas foram selecionadas com base na média dos registros de três lactações e os machos, a partir da média da produção de 100 filhas.

A seleção de fêmeas no núcleo foi feita com base na produção à primeira lactação. Para aumentar a acurácia na seleção de suas progênies, as novas lactações produzidas pelas doadoras foram adicionadas aos registros anteriores. Da mesma forma, foi permitido aos reprodutores que produziram progênies fora do núcleo incluírem as informações do teste dessas progênies nas informações obtidas dentro do núcleo para esses reprodutores.

Os valores gênicos $\left(a_{j}\right)$ das progênies foram gerados de uma distribuição normal com média zero para o genótipo médio dos pais e variância igual a $1 / 2 \sigma^{2}$ a (1-F), em que $\sigma^{2}$ a é a variância genética aditiva da característica na geração-base e F é o coeficiente de endogamia médio dos pais. $\mathrm{O}$ valor gênico de machos e fêmeas foi gerado separadamente. Os registros das fêmeas foram gerados da soma do valor genético aos efeitos ambientes normalmente distribuídos. 
A seleção baseou-se nos valores gênicos estimados por um modelo animal BLUP. O modelo utilizado foi:

$Y_{i j k}=g_{i}+a_{j}+P_{j}+e_{j k}$, em que:

$\mathrm{Y}_{\mathrm{ijk}}=$ observação;

$\mathrm{g}_{\mathrm{i}}=$ média da geração;

$\mathrm{a}_{\mathrm{j}}=$ valor gênico do animal;

$\mathrm{P}_{\mathrm{j}}=$ efeito ambiente permanente;

$\mathrm{e}_{\mathrm{jk}}=$ efeito residual.

Os valores gênicos incluíram os efeitos de grupos genéticos. Os grupos foram formados apenas para os animais que tinham pais desconhecidos (geração-base e progênies fora do núcleo), segundo as regras apresentadas por Westell et al. (1988). As equações do modelo misto foram resolvidas usando-se um procedimento iterativo.

Os efeitos sobre $\mathrm{F}$ foram determinados da probabilidade de seleção conjunta de irmãos (Falconer, 1989) e expressos pela taxa de endogamia $(\Delta \mathrm{F})$, a partir da equação:

$\Delta \mathrm{F}=\frac{\mathrm{F}_{1}-\mathrm{F}_{\mathrm{t}-1}}{1-\mathrm{F}_{\mathrm{t}-1}}$, em que:

$F_{t}$ é a média do coeficiente de endogamia dos animais na geração t. A taxa de endogamia total foi calculada como a média das taxas de endogamia da segunda à oitava gerações em cada esquema alternativo. Para o cálculo da taxa anual de endogamia, foi utilizado o intervalo de gerações assumido em cada esquema.

Os ganhos genéticos médios e as taxas médias de endogamia obtidos para cada esquema alternativo dentro de cada nível de herdabilidade, expressos em porcentagem de desvio-padrão fenotípico (\%UDPF), foram analisados pelo método dos quadrados mínimos ponderados, utilizando-se os procedimentos disponíveis nos programas $\mathrm{SAS} \otimes$, de acordo com um fatorial $3 \times 3 \times 2 \times 2$, correspondente, respectivamente, ao tamanho da população, à estratégia de acasalamento, ao número de progênies MOET nascidas e ao número de reprodutores selecionados por família de irmãos completos (Tab. 1).

\section{RESULTADOS E DISCUSSÃO}

$\mathrm{Na}$ Tab. 2 são apresentados os resultados para o ganho genético e a taxa de endogamia. Como esperado, observou-se que os ganhos genéticos foram maiores quanto maior $o$ valor da herdabilidade considerado, independente do esquema alternativo simulado, pois, neste caso, a maior parte da variação fenotípica na característica é devido aos efeitos genéticos aditivos (Falconer, 1989). Para a taxa de endogamia, menores valores foram observados quanto maior o valor de herdabilidade considerado. Este resultado está associado à diminuição na probabilidade de seleção de indivíduos de uma mesma família e à maior ênfase na seleção individual (Woolliams, 1989; Woolliams e Wilmut, 1989).

Tabela 2. Médias e respectivos desvios-padrão do ganho genético anual (\%UDPF) e da taxa anual de endogamia (\%UDP), obtidos dos resultados dos esquemas alternativos, em função das herdabilidades consideradas

Herdabilidade Ganho genético Taxa de endogamia

\begin{tabular}{lll}
\hline 0,25 & $6,98 \pm 1,17$ & $1,56 \pm 0,40$ \\
0,35 & $8,47 \pm 1,38$ & $1,47 \pm 0,42$
\end{tabular}

$0,35 \quad 8,47 \pm 1,38 \quad 1,47 \pm 0,42$

$0,45 \quad 10,05 \pm 1,69 \quad 1,38 \pm 0,39$

$\%$ UDPF $=$ porcentagem de unidades de desvio-padrão fenotípico; \%UDP = porcentagem de desvio-padrão.

As análises de variância do ganho genético e da taxa de endogamia em função dos fatores utilizados na definição dos esquemas alternativos de núcleo MOET são apresentadas nas Tab. 3 e 4, de acordo com os valores de herdabilidade considerados.

O tamanho da população $(\mathrm{N})$, a estratégia de acasalamento (A), o número de progênies MOET nascidas $(\mathrm{P})$ e o número de reprodutores selecionados por grupo de irmãos completos $(\mathrm{R})$ tiveram efeito significativo $(\mathrm{P}<0,05)$ sobre $\mathrm{O}$ ganho genético, em qualquer nível de herdabilidade considerado (Tab. 3). As interações $\mathrm{N} \times \mathrm{A}, \mathrm{N} \times \mathrm{R}$ e $\mathrm{P} \times \mathrm{A}$ também foram significativas. $\mathrm{A}$ interação $\mathrm{A} \times \mathrm{R}$ foi significativa apenas para as herdabilidades de 0,25 e 0,35. 
Tabela 3. Análise de variância do ganho genético anual observado, em função dos fatores utilizados na definição de cada esquema alternativo de núcleo

\begin{tabular}{|c|c|c|c|c|}
\hline \multirow{3}{*}{$\begin{array}{l}\text { Fonte de } \\
\text { variação }\end{array}$} & \multirow[b]{3}{*}{ GL } & \multicolumn{3}{|c|}{ Herdabilidade } \\
\hline & & 0,25 & 0,35 & 0,45 \\
\hline & & $\mathrm{QM}$ & QM & QM \\
\hline $\mathrm{N}$ & 2 & $141,8096^{*}$ & $223,1467 *$ & $341,7091^{*}$ \\
\hline $\mathrm{P}$ & 1 & $314,1229 *$ & $551,5628 *$ & $804,2055^{*}$ \\
\hline $\mathrm{A}$ & 2 & $547,2383^{*}$ & $834,8465^{*}$ & $1364,4702^{*}$ \\
\hline $\mathrm{R}$ & 1 & $104,7652 *$ & $139,8907^{*}$ & $159,7518^{*}$ \\
\hline $\mathrm{N} \times \mathrm{P}$ & 2 & $0,1511 \mathrm{~ns}$ & $1,8024 \mathrm{~ns}$ & $0,1268^{\mathrm{ns}}$ \\
\hline $\mathrm{N} \times \mathrm{A}$ & 4 & $10,8269^{*}$ & $26,4883^{*}$ & $37,5732 *$ \\
\hline $\mathrm{N} \times \mathrm{R}$ & 2 & $12,9360 *$ & $9,4366^{*}$ & $15,2657^{*}$ \\
\hline $\mathrm{P} \times \mathrm{A}$ & 2 & $86,6110^{*}$ & $144,9627^{*}$ & $202,0686^{*}$ \\
\hline $\mathrm{P} \times \mathrm{R}$ & 1 & $0,0480 \mathrm{~ns}$ & $0,5040 \mathrm{~ns}$ & $0,1957 \mathrm{~ns}$ \\
\hline $\mathrm{A} \times \mathrm{R}$ & 2 & $4,0402 *$ & $6,0776^{*}$ & $3,3579 \mathrm{~ns}$ \\
\hline Resíduo & 1778 & 1,2484 & 1,6330 & 1,8279 \\
\hline
\end{tabular}

Tabela 4. Análise de variância da taxa anual de endogamia observada em função dos fatores utilizados na definição de cada esquema alternativo de núcleo

\begin{tabular}{|c|c|c|c|c|}
\hline \multirow{3}{*}{$\begin{array}{l}\text { Fonte de } \\
\text { variação }\end{array}$} & \multirow[b]{3}{*}{ GL } & \multicolumn{3}{|c|}{ Herdabilidade } \\
\hline & & 0,25 & 0,35 & 0,45 \\
\hline & & QM & QM & QM \\
\hline$\overline{\mathrm{N}}$ & 2 & $95,7391 *$ & $83,9848^{*}$ & $75,2229 *$ \\
\hline $\mathrm{P}$ & 1 & $24,7163 *$ & $19,8208 *$ & $16,7129 *$ \\
\hline A & 2 & $14,0996^{*}$ & $16,8186^{*}$ & $11,7202 *$ \\
\hline $\mathrm{R}$ & 1 & $66,4186^{*}$ & $67,9642 *$ & $60,3406^{*}$ \\
\hline $\mathrm{N} \times \mathrm{P}$ & 2 & $0,4081 *$ & $0,2494^{*}$ & $0,2787^{*}$ \\
\hline $\mathrm{N} \times \mathrm{A}$ & 4 & $0,7750 *$ & $0,8760^{*}$ & $0,7127^{*}$ \\
\hline $\mathrm{N} \times \mathrm{R}$ & 2 & $0,7163^{*}$ & $1,2882 *$ & $1,8346^{*}$ \\
\hline $\mathrm{P} \times \mathrm{A}$ & 2 & $6,2490 *$ & $4,9605^{*}$ & $4,2378 *$ \\
\hline $\mathrm{P} \times \mathrm{R}$ & 1 & $0,0541 \mathrm{~ns}$ & $0,0118 \mathrm{~ns}$ & $0,0817 \mathrm{~ns}$ \\
\hline $\mathrm{A} \times \mathrm{R}$ & 2 & $0,0846 \mathrm{~ns}$ & $0,0444 \mathrm{~ns}$ & $0,0122 \mathrm{~ns}$ \\
\hline Resíduo & 1778 & 0,1350 & 0,0792 & 0,0708 \\
\hline
\end{tabular}

Com relação à taxa de endogamia, foram verificados os efeitos significativos de todos os fatores e das interações $\mathrm{N} \times \mathrm{P}, \mathrm{N} \times \mathrm{A}, \mathrm{P} \times \mathrm{R}$ e $\mathrm{P} \times \mathrm{A}$, em todos os níveis de herdabilidade considerados. Em função da significância das interações duplas, os efeitos dos fatores simples sobre a média do ganho genético não serão discutidos. As mudanças na taxa de endogamia só serão discutidas para as interações significativas para o ganho genético, principal objetivo deste trabalho.

Na Tab. 5 observam-se maiores ganhos genéticos anuais para a interação entre tamanho do núcleo e estratégia de acasalamento $(\mathrm{N} \times \mathrm{A})$, quando se utilizou o núcleo com maior número de doadoras por reprodutor, associado à estratégia fatorial II. $\mathrm{O}$ aumento no número de doadoras por reprodutor resultou em maior número de progênies por reprodutor, o que permitiu maior utilização da variação dentro de família e minimizou os prejuízos na intensidade e na acurácia de seleção decorrentes da redução no tamanho da família na estratégia fatorial de acasalamento. A estratégia fatorial de acasalamento possibilita também a seleção para os efeitos genéticos não aditivos, propiciando ganhos genéticos ainda maiores (Ruane e Thompson, 1991; Strandén et al., 1991; Dekkers, 1992).

Quanto à taxa de endogamia, maiores valores foram observados para a combinação do maior tamanho de núcleo à estratégia fatorial II de acasalamento. Provavelmente, o aumento no número de doadoras por reprodutor, associado à utilização da estratégia fatorial de acasalamento, resultou em maior número de progênies, e grupos de meio-irmãos, por reprodutor, aumentando a acurácia da seleção e a contribuição genética de cada reprodutor para formação da geração seguinte, uma vez que a seleção nesses esquemas baseia-se nas informações de família (Woolliams, 1989). O aumento na possibilidade de seleção de fêmeas irmãs completas, já que a seleção baseou-se nos índices de família e a seleção de machos por grupos de irmãos completos foi limitada a um ou dois, pode também ter contribuído para o aumento na taxa de endogamia.

Como as características reprodutivas são muito sensíveis ao aumento no coeficiente de endogamia, a depressão endogâmica poderá prejudicar a eficiência do núcleo MOET, diminuindo o número de embriões viáveis por doadora (Woolliams, 1989; Keller et al., 1990; Strandén et al., 1991). Faz-se imprescindível, portanto, procurar um balanço entre ganho genético e taxa de endogamia. Ruane (1991) e Ruane e Thompson (1991) recomendaram que o número de progênies MOET selecionado por doadora fosse limitado a um número ótimo a ser determinado para cada combinação. 
Seleção para produção de leite...

Tabela 5. Média dos quadrados mínimos do ganho genético anual $(\Delta \mathrm{G})$ e da taxa anual de endogamia $(\Delta \mathrm{F})$ em função da interação $\mathrm{N} \times \mathrm{A}$, para os três níveis de herdabilidade considerados

\begin{tabular}{|c|c|c|c|c|c|c|c|}
\hline \multirow{3}{*}{$\begin{array}{l}\text { Estratégia de } \\
\text { acasalamento (A) }\end{array}$} & & \multicolumn{6}{|c|}{ Tamanho do núcleo $(\mathrm{N})$} \\
\hline & & \multicolumn{2}{|c|}{$16 / 8(2)$} & \multicolumn{2}{|c|}{$16 / 4(4)$} & \multicolumn{2}{|c|}{$32 / 4(8)$} \\
\hline & & $\Delta \mathrm{G}$ & $\Delta \mathrm{F}$ & $\Delta \mathrm{G}$ & $\Delta \mathrm{F}$ & $\Delta \mathrm{G}$ & $\Delta \mathrm{F}$ \\
\hline \multirow{3}{*}{$\mathrm{h}^{2}=0,25$} & Hierárquica & 5,1166 & 0,8727 & 5,7706 & 1,7116 & 6,4672 & 1,6250 \\
\hline & Fatorial I & 6,3310 & 1,0409 & 6,3166 & 1,7740 & 7,0850 & 1,6483 \\
\hline & Fatorial II & 7,5144 & 1,3092 & 7,3900 & 1,9672 & 8,1538 & 1,8254 \\
\hline \multirow{3}{*}{$h^{2}=0,35$} & Hierárquica & 6,5687 & 0,8238 & 7,2180 & 1,5799 & 8,3926 & 1,5591 \\
\hline & Fatorial I & 7,6629 & 1,0139 & 8,0939 & 1,1720 & 8,8575 & 1,5638 \\
\hline & Fatorial II & 9,6806 & 1,2757 & 9,3369 & 1,9146 & 10,1344 & 1,7564 \\
\hline \multirow{3}{*}{$h^{2}=0,45$} & Hierárquica & 7,5666 & 0,7887 & 8,5706 & 1,4963 & 9,8518 & 1,4645 \\
\hline & Fatorial I & 9,0921 & 0,9388 & 9,8121 & 1,6170 & 10,6062 & 1,5390 \\
\hline & Fatorial II & 11,5020 & 1,1946 & 11,3076 & 1,7442 & 12,1582 & 1,6451 \\
\hline
\end{tabular}

O esquema alternativo em que uma população de menor tamanho (16/8), utilizando a estratégia fatorial II de acasalamento, foi aquele em que elevados ganhos genéticos a baixas taxas de endogamia foram alcançados (Tab. 5). Este resultado pode ser atribuído à menor seleção dentro de família, devido ao menor número de doadoras por reprodutor, quando a estratégia fatorial II de acasalamento, que propicia maior acurácia e intensidade de seleção, foi praticada.

Dois aspectos devem ainda ser considerados com relação a essa interação: de núcleos menores obtém-se maior salto genético inicial na geraçãobase (Nicholas e Smith, 1983; Ruane e Thompson, 1991), e o aumento no número de reprodutores por doadora, na estratégia fatorial de acasalamento, pode conduzir ao aumento no intervalo de gerações e à conseqüente redução no ganho genético anual.
Na Tab. 6 é apresentada a interação entre tamanho do núcleo e número de reprodutores selecionados por família de irmãos completos $(\mathrm{N} \times \mathrm{R})$. Verificou-se a superioridade do esquema com maior número de doadoras por reprodutor associado à maior seleção de reprodutores dentro de grupo de irmãos completos. A exploração da variação dentro de família de irmãos completos e o aumento do tamanho efetivo da população, propiciados pelo aumento no número de reprodutores selecionados de grupo de irmãos completos, foram potencializados pelo aumento no número de fêmeas por reprodutor e, conseqüentemente, no tamanho das famílias de irmãos completos, com resultados sobre a acurácia e a intensidade de seleção (Woolliams, 1989; Ruane, 1991; Ruane e Thompson, 1991).

Tabela 6. Média dos quadrados mínimos do ganho genético anual $(\Delta \mathrm{G})$ e da taxa anual de endogamia $(\Delta \mathrm{F})$ em função da interação $\mathrm{N} \times \mathrm{R}$, para os três níveis de herdabilidade considerados

\begin{tabular}{|c|c|c|c|c|c|c|c|}
\hline \multirow{3}{*}{$\begin{array}{l}\text { Número de reprodutores } \\
\text { selecionados/família de IC (R) }\end{array}$} & & \multicolumn{6}{|c|}{ Tamanho do núcleo $(\mathrm{N})$} \\
\hline & & \multicolumn{2}{|c|}{$16 / 8(2)$} & \multicolumn{2}{|c|}{$16 / 4(4)$} & \multicolumn{2}{|c|}{$32 / 4(8)$} \\
\hline & & $\Delta \mathrm{G}$ & $\Delta \mathrm{F}$ & $\Delta \mathrm{G}$ & $\Delta \mathrm{F}$ & $\Delta \mathrm{G}$ & $\Delta \mathrm{F}$ \\
\hline \multirow{2}{*}{$\mathrm{h}^{2}=0,25$} & 1 & 6,2468 & 1,2304 & 6,1442 & 2,0426 & 6,9336 & 1,8946 \\
\hline & 2 & 6,3945 & 0,9181 & 6,8406 & 1,5926 & 7,5371 & 1,5045 \\
\hline \multirow{2}{*}{$\mathrm{h}^{2}=0,35$} & 1 & 7,8262 & 1,1824 & 7,9173 & 1,9685 & 8,7353 & 1,8285 \\
\hline & 2 & 8,1152 & 0,8932 & 8,5153 & 1,4959 & 8,5210 & 1,4244 \\
\hline \multirow{2}{*}{$\mathrm{h}^{2}=0,45$} & 1 & 9,2243 & 1,0982 & 9,6394 & 1,8531 & 10,3983 & 1,7407 \\
\hline & 2 & 9,5495 & 0,8498 & 10,1541 & 1,3853 & 11,3459 & 1,3583 \\
\hline
\end{tabular}

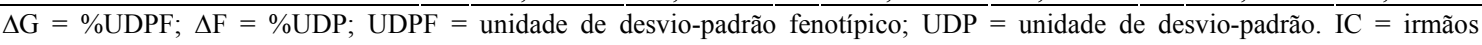
completos 
A taxa de endogamia alcançou menores valores quando maior tamanho do núcleo (32/4) esteve associado à seleção de um número maior de reprodutores por grupo de irmãos completos. Esse esquema conduziu a ganhos genéticos maiores, com taxas de endogamia menores, do que aqueles em que se selecionou apenas um reprodutor por grupo de irmãos completos, resultado importante quando considerado o balanço entre ganho genético e taxa de endogamia. $\mathrm{O}$ aumento no número de grupos de irmãos completos, possivelmente, minimizou os efeitos prejudiciais da seleção de mais de um reprodutor por grupo de irmãos completos sobre o coeficiente de endogamia a cada geração, em função da utilização da variação dentro desses grupos (Nicholas e Smith, 1983; Woolliams, 1989). Os aspectos relativos aos custos do aumento da população e à redução do salto genético inicial também devem, no entanto, ser considerados.
$\mathrm{Na}$ Tab. 7 a restrição desse programa de simulação, que assume a produção de pelo menos uma progênie feminina por acasalamento, explica a igualdade nos resultados dos ganhos genéticos e das taxas de endogamia para a interação entre número de progênies MOET nascidas e estratégia de acasalamento $(\mathrm{P} \times \mathrm{A})$. Nesse caso, quando quatro progênies foram obtidas por acasalamento, a condição de produção de pelo menos uma progênie feminina por doadora fez com que todas as quatro progênies dos acasalamentos fatorial II fossem femininas, apesar da probabilidade de $50 \%$ para o nascimento de indivíduos de cada sexo. Isso fez com que esse resultado se igualasse àquele obtido quando o número de progênies por doadora foi igual a oito, sendo uma progênie masculina e outra feminina por acasalamento, portanto, quatro progênies femininas por doadora.

Tabela 7. Média dos quadrados mínimos do ganho genético anual $(\Delta \mathrm{G})$ e da taxa anual de endogamia $(\Delta \mathrm{F})$ em função da interação $\mathrm{P} \times \mathrm{A}$, para os três níveis de herdabilidade considerados

\begin{tabular}{|c|c|c|c|c|c|c|c|}
\hline \multirow{3}{*}{$\begin{array}{l}\text { Número de progênies MOET } \\
\text { nascidas por acasalamento (P) }\end{array}$} & & \multicolumn{6}{|c|}{ Estratégia de acasalamento (A) } \\
\hline & & \multicolumn{2}{|c|}{ Hierárquica } & \multicolumn{2}{|c|}{ Fatorial I } & \multicolumn{2}{|c|}{ Fatorial II } \\
\hline & & $\Delta \mathrm{G}$ & $\Delta \mathrm{F}$ & $\Delta \mathrm{G}$ & $\Delta \mathrm{F}$ & $\Delta \mathrm{G}$ & $\Delta \mathrm{F}$ \\
\hline \multirow{2}{*}{$\mathrm{h}^{2}=0,25$} & 4 & 5,0488 & 1,2179 & 6,0574 & 1,3212 & 7,6890 & 1,7008 \\
\hline & 8 & 6,5208 & 1,5883 & 7,0976 & 1,6542 & 7,6890 & 1,7008 \\
\hline \multirow{2}{*}{$\mathrm{h}^{2}=0,35$} & 4 & 6,4542 & 1,1665 & 7,4830 & 1,2662 & 9,7173 & 1,6489 \\
\hline & 8 & 8,3320 & 1,4754 & 8,9265 & 1,5869 & 9,7173 & 1,6489 \\
\hline \multirow{2}{*}{$\mathrm{h}^{2}=0,45$} & 4 & 7,6192 & 1,0953 & 8,8754 & 1,2304 & 11,6569 & 1,5280 \\
\hline & 8 & 9,7068 & 1,4043 & 10,7982 & 1,4995 & 11,6559 & 1,5280 \\
\hline
\end{tabular}

$\triangle \mathrm{G}=\% \mathrm{UDPF} ; \Delta \mathrm{F}=\% \mathrm{UDP} ; \mathrm{UDPF}=$ unidade de desvio-padrão fenotípico; UDP $=$ unidade de desvio-padrão.

A superioridade das combinações do número maior de progênies MOET nascidas com a estratégia fatorial II, quanto ao ganho genético, está associada à estimativa dos efeitos genéticos não aditivos e à maior exploração da variância genética existente na característica, potencializadas pelo aumento no número de progênies nascidas, o que possibilitou maior acurácia e intensidade de seleção. Esse aspecto é de extrema relevância, pois se verificou grande variabilidade no número de embriões viáveis obtidos por doadora por superovulação nas raças zebuínas (Coelho et al., 1989; Coelho e Azevedo, 1991; Penna et al., 1998), o que levaria à necessidade de novas superovulações para proceder às avaliações genéticas e, conseqüentemente, ao aumento no intervalo de gerações, com prejuízos para a taxa de progresso genético (Ruane, 1991).

Quando se considerou a endogamia, o aumento no número de progênies MOET por doadora, associado à estratégia fatorial II de acasalamento, propiciou maior seleção de indivíduos de uma mesma família, aparentados pelo lado materno, conduzindo ao aumento da taxa de endogamia (Strandén et al., 1991). Quando se considerou o IG de 4,15 anos nas simulações com a estratégia fatorial II de acasalamento, verificou-se decréscimo da taxa de endogamia, resultando em valores abaixo daqueles obtidos nos esquemas hierárquicos de acasalamento. Para esse valor de IG, a média observada da taxa anual de endogamia, para todas as combinações nas quais 
se praticou a estratégia fatorial II de acasalamento, foi de 1,74\%UDP e, quando se praticou a estratégia hierárquica, foi de $1,76 \%$ UDP.

Quando se considera o balanço entre ganho genético e taxa de endogamia, o esquema mais indicado seria a utilização da estratégia fatorial I de acasalamento e a produção de até quatro progênies por reprodutor para proceder à avaliação genética.

$\mathrm{Na}$ Tab. 8 são mostrados os resultados da interação estratégia de acasalamento versus número de reprodutores selecionados por grupo de irmãos completos $(\mathrm{A} \times \mathrm{R})$. Os melhores resultados para o ganho genético foram obtidos quando a estratégia fatorial II de acasalamento e o maior número de reprodutores foram selecionados por grupo de irmãos completos. A associação entre utilização da variação genética aditiva e de dominância, cuja estimativa foi permitida pela estratégia fatorial de acasalamento, e da variação existente dentro de família, quando da seleção de dois irmãos de um mesmo grupo de irmãos completos, propiciou maior intensidade de seleção, levando a maiores ganhos genéticos nessa interação (Ruane, 1991; Ruane e Thompson, 1991; Villanueva e Simm, 1994).

Tabela 8. Média dos quadrados mínimos do ganho genético anual $(\Delta \mathrm{G})$ em função da interação AxR, para os três níveis de herdabilidade considerados

\begin{tabular}{lcccc}
\hline $\begin{array}{l}\text { Número de reprodutores } \\
\text { selecionados/família de IC (R) }\end{array}$ & & \multicolumn{3}{c}{ Estratégia de acasalamento (A) } \\
\cline { 3 - 5 } $\mathrm{h}^{2}=0,25$ & 1 & 5,5483 & Fatorial I & Fatorial II \\
& 2 & 6,0212 & 6,4158 & 7,3605 \\
$\mathrm{~h}^{2}=0,35$ & 1 & 7,2267 & 6,7392 & 8,0117 \\
& 2 & 7,5595 & 7,8954 & 9,3567 \\
& 1 & 8,4457 & 8,5142 & 10,0779 \\
$\mathrm{~h}^{2}=0,45$ & 2 & 8,8804 & 9,5258 & 11,2906 \\
& & & 10,1478 & 12,0213 \\
\hline
\end{tabular}

IC = irmãos completos.

Na prática, a chance de se produzir pelo menos dois irmãos completos por superovulação é menor com a estratégia fatorial. Segundo Ruane (1988) e Ruane (1991), esse fato poderia levar à redução na intensidade de seleção de machos para constituírem a nova geração. Dekkers (1992) concluiu que a intensidade de seleção de fêmeas seria prejudicada, pois o número de doadoras no núcleo é maior do que o de reprodutores e não há restrição à seleção de fêmeas por grupo de irmãos completos. De acordo com Woolliams e Wilmut (1989), a redução no número de irmãs completas e meiairmãs levaria também à menor acurácia na estimativa do valor genético dos machos e, portanto, a menores ganhos genéticos.

O coeficiente de endogamia não foi influenciado pela interação $\mathrm{A} \times \mathrm{R}$, portanto, o aumento no número de reprodutores selecionados por grupo de irmãos completos, associado às diferentes estratégias de acasalamento, não alterou a taxa de endogamia nos esquemas alternativos simulados.

Nas Tab. 5, 6 e 7, ao se confrontarem os resultados obtidos para o ganho genético e para a taxa de endogamia, verificou-se que nem sempre os esquemas que produziram os maiores ganhos genéticos produziram, concomitantemente, as menores taxas de endogamia. Isso pode ser atribuído ao fato de que os esquemas que propiciaram maiores acurácia e intensidade de seleção propiciaram, também, maior seleção de indivíduos aparentados e, portanto, aumento no coeficiente médio de endogamia, como discutido por Keller et al. (1990). Dentre os esquemas que alcançaram sucesso em produzir alto ganho genético, a baixa taxa de endogamia, balanço imprescindível a um esquema de melhoramento, ressalta-se aquele em que se assumiu um núcleo de maior tamanho, com maior número de doadoras por reprodutor, associado à seleção de dois reprodutores por família de irmãos completos.

Alguns autores têm apresentado diversas sugestões para evitar o aumento no coeficiente de endogamia em núcleos MOET. Smith (1988) propôs que os núcleos estivessem continuamente abertos à introdução de indivíduos geneticamente superiores de outros rebanhos. Toro et al. (1991) discutiram o planejamento de acasalamentos 
como forma de evitar que indivíduos aparentados se acasalem e de minimizar a taxa de endogamia no núcleo. Ambas as sugestões merecem consideração diante dos resultados apresentados para a taxa de endogamia neste trabalho. Outras alternativas que permitam minimizar a taxa de endogamia no núcleo foram apontadas por Colleau (1998), dentre as quais o autor ressalta a técnica da seleção assistida por marcadores.

A utilização de um modelo estocástico, que considera a redução na variância genética decorrente do desequilíbrio de ligação induzido pela seleção (Bulmer, 1971), e o aumento no coeficiente de endogamia em núcleo fechado (Smith, 1988) conferem maior acurácia aos resultados obtidos nas simulações. Desse modo, este estudo fornece um bom indicativo a respeito das alternativas a serem adotadas (Verrier et al., 1990; Ruane e Thompson, 1991).

Deve-se salientar a importância da redução no IG para a obtenção de taxas de ganho genético expressivas em núcleos MOET de seleção. A idade ao primeiro parto tem apresentado variação de 37 a 54 meses em raças zebuínas (Nájera, 1990; Bergmann, 1993); valor maior do que aquele utilizado nas simulações (25 e 32 meses), o que significa intervalo de gerações mais longo e prejuízos ao ganho genético em núcleos MOET. Em vários rebanhos brasileiros, no entanto, as estimativas da idade à puberdade indicam a possibilidade de precocidade reprodutiva dos animais zebuínos e de IG satisfatório para a condução de programas de melhoramento baseados em núcleos MOET (Cézar e Euclides Filho, 1996; Bergmann et al., 1998).

\section{CONCLUSÕES}

Pode-se concluir que a utilização da estratégia fatorial de acasalamento propiciará maiores ganhos genéticos a baixas taxas de endogamia quando o núcleo possuir menor número de doadoras por reprodutor ou quando no máximo quatro progênies por acasalamento forem produzidas. A utilização da estratégia fatorial de acasalamento, quando selecionados dois reprodutores por grupo de irmãos completos, produz maiores ganhos genéticos sem acréscimo na taxa de endogamia. Quando estabelecido núcleo com maior número de doadoras por reprodutor, os resultados indicam que melhores balanços entre ganho genético e taxa de endogamia serão obtidos quando da seleção de dois reprodutores por grupo de irmãos completos. Torna-se de fundamental importância a produção de número mínimo de progênies por acasalamento em menor intervalo de tempo para garantir o sucesso dos esquemas de seleção em núcleos MOET.

\section{REFERÊNCIAS BIBLIOGRÁFICAS}

BERGMANN, J.A.G.; GRESSLER, S.L.; PEREIRA, C.S. et al. Avaliação de fatores genéticos e de ambiente sobre diferentes características reprodutivas de fêmeas da raça Nelore em regime de estação de monta restrita. Arq. Bras. Med. Vet. Zootec., v.50, p.633 - 645, 1998.

BERGMANN, J.A.G. Melhoramento genético da eficiência reprodutiva em bovinos de corte. In: CONGRESSO BRASILEIRO DE REPRODUÇÃO ANIMAL, 10., 1993, Belo Horizonte. Anais... Belo Horizonte: CBRA, 1993. p.70-86.

BULMER, M.G. The effect of selection on genetic variability. Am. Natur., v.105, p.202-211, 1971.

CEZAR, I.M.; EUCLIDES FILHO, K. Novilho precoce: reflexos na eficiência e economicidade do sistema de produção. Campo Grande: EMBRAPA-CNPGC, 1996. 31p. (Documentos, 66).

COELHO, E.N.; AZEVEDO, N.A. Particularidades da transferência de embriões zebuínos. In: CONGRESSO BRASILEIRO DE REPRODUÇÃO ANIMAL, 9., 1991, Belo Horizonte. Anais... Belo Horizonte : CBRA, 1991. 470p.

COELHO, S.G.; FREITAS, C.; CHOW, L.A. Transferência de embriões de raças zebuínas. Arq. Bras. Med. Vet. Zootec., v.41, p.459-460, 1989.

COLLEAU, J.J. Relative economic efficiency of embryo transfer and marker selection in dairy cattle selection nuclei. In: WORLD CONGRESS OF GENETICS APPLIED TO LIVESTOCK PRODUCTION, 6., Armidale, 1998. Proceedings... Armidale : WCGALP, 1998. 
DEKKERS, J.C.M. Structure of breeding programs to capitalize on reproductive technology for genetic improvement. J. Dairy Sci., v.75, p.2880-2891, 1992.

FALCONER, D.S. Introduction to quantitative genetics. 3.ed. New York : Longman, 1989. $340 \mathrm{p}$.

HINKS, C.J.M. The use of centralized breeding schemes in dairy cattle improvement. Anim. Breed. Abstr., v.46, p.291-297, 1978. (Review Article).

KELLER, D.S.; TEEPKER, G. Effect of variability in response to superovulation on donor cow selection differentials in nucleus breeding schemes. J. Dairy Sci., v.73, p.549-554, 1990.

LÔBO, R.N.B.; MADALENA, F.E.; VIEIRA, A.R. Average estimates of genetic parameters for beef and dairy cattle in tropical regions. Anim. Breed. Abstr., v.68, p.433-462, 2000.

MADALENA, F.E. A note on the effect of variation of lactation length on the efficiency of tropical cattle selection for milk yield. Theor. Appl. Gen., v.76, p.830-834, 1988.

NÁJERA, A.J.M. Efeitos genéticos e não genéticos sobre caracteristicas reprodutivas $e$ ponderais de duas populações de bovinos da raça Nelore. Universidade Federal de Minas Gerais, 1988. 89f. Dissertação (Mestrado) Escola de Veterinária, Universidade Federal de Minas Gerais, Belo Horizonte.

NICHOLAS, F.W.; SMITH, C. Increased rates of genetic change in dairy cattle by embryo transfer and splitting. Anim. Prod., v.36, p.341353,1983

PENNA, V.M.; MADALENA, F.E.; ALVIM, M.T.T. Open MOET selection nucleus in Guzerá. In: WORLD CONGRESS OF GENETICS APPLIED TO LIVESTOCK PRODUCTION, 6., 1998, Armidale. Proceedings... Armidale: WCGALP, 1998. p.439.

RUANE, J. Review of the use of embryo transfer in the genetic improvement of dairy cattle. Anim. Breed. Abstr., v.56, p.437-446, 1988.

RUANE, J. The importance of family sizes in an adult multiple ovulation and embryo transfer
(MOET) nucleus breeding schemes in dairy cattle. Anim. Prod., v.52, p.33-47, 1991.

RUANE, J.; THOMPSON, R. Comparison of simulated and theoretical results in an adult MOET nucleus schemes for dairy cattle. Liv. Prod. Sci., v.28, p.1-20, 1991.

SACCO, R.E.; BAKER, J.F.; CARTWRIGHT, T.C. Production characters of primiparous females of a five-breed diallel. J. Anim. Sci., v.64, p.1612-1618, 1987.

SEIDEL Jr., G.E. Applications of embryo transfer and related technologies to cattle. $J$. Dairy Sci., v.67, p.2786-2796, 1984.

SMITH, C. Efficiency of animal testing schemes. Biometrics, v.16, p.408-415, 1960.

SMITH, C. Genetic improvement of livestock using nucleus-breeding units. World Anim. Rev., v.65, p.2-10, 1988.

STRANDÉN, I.; MÄKI-TANILA, A.; MÄNTYSAARI, E.A. Genetic progress and rate of inbreeding in a closed adult MOET nucleus under different mating strategies and heritabilities. J. Anim. Breed. Gen., v.108, p.401411, 1991.

TORO, M.A.; SILIÓ, L.; PEREZ-ENCISO, M. A note on the use of male selection in closed MOET selection. Anim. Prod., v.53, p.403-406, 1991.

VERRIER, E.; COLLEAU, J.J.; FOULLEY, J.L. Predicting cumulated response to directional selection in finite panmitic populations. Theor. Appl. Gen., v.79, p.833-840, 1990.

VILLANUEVA, B., SIMM, G. The use and value of embryo manipulation techniques in animal breeding. In: WCGALP, 5., Guelph, 1994. Proceedings... Guelph: WCGALP, 1994.

WESTELL, C.W., QUAAS, R.L., VAN VLECK, L.D. Genetic groups in an animal model. J. Dairy Sci., v.71, p.1310-1318, 1988.

WOOLLIAMS, J.A. Modifications to MOET nucleus breeding schemes to improve rates of genetic progress and decrease rates of inbreeding in dairy cattle. Anim. Prod., v.49, p.1-14, 1989.

WOOLLIAMS, J.A., WILMUT, I. Embryo manipulation in cattle breeding and production. Anim. Prod., v.48, p.3-30, 1989. 